\title{
Rapid Detection of SARS-CoV-2 Variants of Concern, Including B.1.1.28/P.1, British Columbia, Canada
}

Nancy Matic, ${ }^{1}$ Christopher F. Lowe, ${ }^{1}$ Gordon Ritchie, Aleksandra Stefanovic, Tanya Lawson, Willson Jang, Matthew Young, Winnie Dong, Zabrina L. Brumme, Chanson J. Brumme, Victor Leung, Marc G. Romney

To screen all severe acute respiratory syndrome coronavirus 2-positive samples in Vancouver, British Columbia, Canada, and determine whether they represented variants of concern, we implemented a real-time reverse transcription PCR-based algorithm. We rapidly identified 77 samples with variants: 57 with B.1.1.7, 7 with B.1.351, and an epidemiologic cluster of 13 with B.1.1.28/P.1.

\footnotetext{
$\Lambda$ robust surveillance system for early identification of severe acute respiratory syndrome coronavirus 2 (SARS-CoV-2) variants of concern (VOCs) is of critical public health value. VOCs have demonstrated in vitro evasion of antibody neutralization $(1,2 ;$ W.F. Garcia-Beltran et al., unpub. data, https:/ / www.medrxiv.org/content/10.1101/2021.02.14.21251704v1) and displayed potential for enhanced transmission because of mutations in the spike receptor binding domain (G. Nelson et al., unpub. data, https:/ / www. biorxiv.org/content/10.1101/2021.01.13.426558v1; H. Liu et al., unpub. data, https:/ / www.biorxiv.org/ content/10.1101/2021.02.16.431305v1). Surveillance from the United Kingdom demonstrated a rapid increase in cases during September 2020, attributed to the B.1.1.7 variant, which has become predominant in several other countries (3). A commercial SARSCoV-2 real-time reverse transcription PCR (rRT-PCR) demonstrated dropout of the small $(S)$ gene on B.1.1.7

Author affiliations: St. Paul's Hospital, Vancouver, British Columbia, Canada (N. Matic, C.F. Lowe, G. Ritchie, A. Stefanovic, T. Lawson, W. Jang, M. Young, V. Leung, M.G. Romney); University of British Columbia, Vancouver (N. Matic, C.F. Lowe, G. Ritchie, A. Stefanovic, C.J. Brumme, V. Leung, M.G. Romney); British Columbia Centre for Excellence in HIVIAIDS, Vancouver (W. Dong, Z.L. Brumme, C.J. Brumme); Simon Fraser University, Burnaby, British Columbia, Canada (Z.L. Brumme)
}

DOI: https://doi.org/10.3201/eid2706.210532 because of a deletion mutation within the spike protein (69/70). Subsequently, the European Centre for Disease Prevention and Control proposed use of a specific commercial assay with 3 targets as a surveillance strategy (4).

The B.1.1.28/P.1 variant is an emerging VOC and the predominant strain in certain regions of Brazil. Although uncommon in North America, it has now been detected across several continents. Re-infection of patients with SARS-CoV-2 immunity has raised concerns about resurgence (5). Unlike B.1.1.7, the B.1.1.28/P.1 variant does not possess the $69 / 70$ deletion mutation, highlighting the need for a versatile VOC surveillance strategy.

Given the potential for VOCs to enhance transmission, increase deaths, and possibly evade natural or vaccine-induced immune responses, identifying cases of coronavirus disease (COVID-19) caused by VOCs and monitoring their prevalence is critical. We propose a rapid VOC surveillance strategy that uses multiple rRT-PCRs to screen all samples positive for SARS-CoV-2. This study was approved by the Providence Health Care/University of British Columbia and Simon Fraser University Research Ethics Boards (H20-01055).

\section{The Study}

During January 26-March 1, 2021, the clinical virology laboratory at St. Paul's Hospital, Vancouver, British Columbia, Canada, conducted VOC testing on nasopharyngeal swab and saliva/mouth rinse samples in which SARS-CoV-2 was detected at any cycle threshold $\left(\mathrm{C}_{\mathrm{t}}\right)$ value. SARS-CoV-2 detection was performed by using the LightMix SarbecoV E-gene plus EAV control assay (TIB

${ }^{1}$ These first authors contributed equally to this article. 
Molbiol, https://www.tib-molbiol.de), with the MagNA Pure Compact or MagNA Pure 96 and LightCycler 480 or with the cobas SARS-CoV-2 Test (Roche Molecular Diagnostics, https://diagnostics.roche.com) on the cobas 6800. VOCs were detected with the VirSNiP SARS-CoV-2 Mutation Assays for strain surveillance (TIB Molbiol), targeting specific spike protein variations (N501Y, delHV69/70, K417N, E484K, V1176F). A laboratorydeveloped test was also developed for N501Y (501F-GCATGTAGAAGTTCAAAAGAAAGT; 501R-TCCT T T A CA A TCA T A TGGTTTCCA; 501YProbe FAM-CACT+T+ATGGT GTTGGTTACCA ACCA-IABkFQ; 501NProbe Cy5-CACT+A+ATGG TGTTGGTTACCAACCA-IAbRQSp)anddelHV69/70 (del6970F-TCAACTCAGGACTTGTTCTTAC; del6970R-TGGTAGGACAGGGTTATCAAAC; wtProbe HEX-TGCTAT+ACATG+TCTCTGGGACCA-IABkFQ; delProbe-TEX615-TGCTAT+CTCTG+GGACCAATGIAbRQSp), in which + denotes locked nucleic acids. Samples were first screened for N501Y, and if detected, we tested subsequent targets to discriminate between the most prevalent VOC within the Vancouver community (B.1.1.7-delHV69/70 and B.1.351-K417N) and newly emerging VOC (B.1.1.28/P.1-V1176F).

For the first presumptive case caused by each VOC, we performed whole-genome sequencing (WGS)
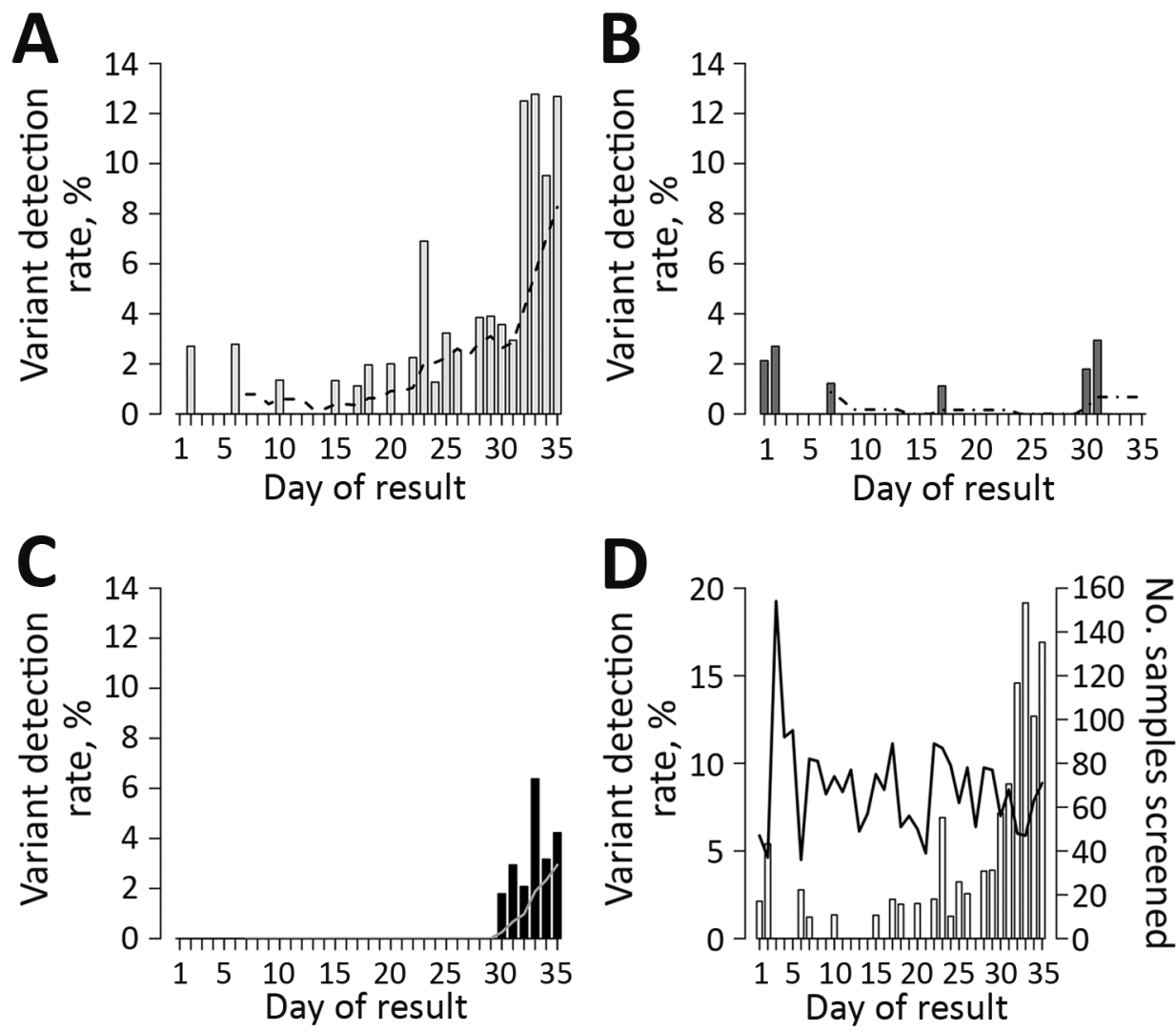

in-house on either the MinION (Oxford Nanopore Technologies, https://nanoporetech.com), using the ARTIC nCOV-2019 sequencing protocol V1 (https:// www.protocols.io/view/ncov-2019-sequencing-protocol-bbmuik6w) by using V3 primers, or on an Illumina MiSeq (https://www.illumina.com) by using a modified ARTIC nCOV-2019 protocol. Accurate base calling of MinION was performed by using GUPPY 3.1.5 and FASTQ files analyzed with BugSeq (https://BugSeq. com). Illumina data were analyzed with the in-house bioinformatics pipeline MiCall (https://github.com/cfelab/MiCall). All presumptive VOCs were subsequently sent to a reference laboratory for confirmatory WGS.

During the study period, 31,833 clinical samples were tested for SARS-CoV-2, and results were positive for 2,618. Of these, 2,430 (92.8\%) underwent testing for the 3 major VOC categories (B.1.1.7, B.1.351, B1.1.28/P.1); 1.6\% (38/2,430) failed to amplify with the N501Y assay, of which $71.0 \%(27 / 38)$ were reported as indeterminate for SARS-CoV-2, reflecting late $C_{t}$ values and presumably low viral loads. From the remaining 2,392 samples, 77 VOCs were identified (57B.1.1.7, 7-B.1.351, and 13-B.1.1.28/P.1). N501Y was not detected in the remaining 2,315 (96.8\%) samples, and they were not sent to the reference laboratory for WGS. The VirSNiP and laboratory-developed PCRs were concordant for all detected VOCs. 
During the study period, VOC detection among diagnostic samples rapidly increased (Figure). Of note, identified VOCs included a large cluster of the B.1.1.28/P.1 variant not previously identified in British Columbia. All B.1.1.28/P.1 variants were initially suspected from the K417N assay, for which PCR products were identified at a lower melting temperature than expected. All suspected B.1.1.28/P.1 variants were confirmed when rescreened by using the V1176F target.

The first presumptive B.1.1.28/P.1 variant identified was confirmed by in-house WGS, detecting the following $S$ gene mutations (characteristic of B.1.1.28/P.1): L18F, T20N, P26S, D138Y, R190S, K417T, E484K, N501Y, D614G, H655Y, T1027I, and V1176F. At the time of publication, the reference laboratory had attempted WGS for $54 / 77$ VOCs, of which $7(12.9 \%)$ samples failed WGS, with original $C_{t}$ values of 24-35. Of the 47 successfully sequenced samples, agreement with PCR was 100\% (38-B.1.1.7, 3-B.1.351, and 6-B.1.1.28/P.1).

\section{Conclusions}

Implementation of a PCR-based algorithm to detect VOCs has enabled our laboratory to rapidly detect new variants that are in the early stages of community transmission. Our protocol enabled detection of VOCs within 24 hours of COVID-19 diagnosis, a marked advantage over sequencing-based surveillance strategies. VOC positivity rate was $3.2 \%$, but detection rates increased markedly over time, as might be expected with exponential growth observed in other countries.

Although B.1.1.28/P.1 had not been previously reported in our region, presence of this variant was suspected when K417N PCR products with a lower melting temperature than wild-type were observed, suggestive of a non-K417N substitution (e.g., K417T mutation in the B.1.1.28/P.1 variant) (VirSNiP SARSCoV-2 Spike K417N package insert; TIB Molbiol, Berlin, Germany). Follow-up testing using the V1176F target supported the presumptive identification of B.1.1.28/P.1, which was subsequently confirmed by WGS. Testing all SARS-CoV-2-positive samples for VOCs enabled rapid detection of a discrete new B.1.1.28/P.1 cluster.

Although WGS has been the primary modality for VOC surveillance, universal sequencing of SARS$\mathrm{CoV}-2$-positive specimens is limited by both laboratory and bioinformatics capacity (6). Because of the volume of VOC testing and the limited capacity for high complexity WGS, turnaround times by WGS may be days to weeks. Since the onset of the CO-
VID-19 pandemic, molecular diagnostics (i.e., PCR) have been increasingly adopted by laboratories to promptly identify SARS-CoV-2, and infrastructure has been established for this testing modality. A PCRbased algorithm for the molecular detection of VOCs could be rapidly adopted, providing almost real-time results to inform infection prevention and control and public health measures $(3,7,8)$. PCR may also be more sensitive because WGS is challenging to perform on samples with low viral loads $\left(C_{t}>30\right)(9)$. Compared with WGS, PCR screening enhanced sensitivity for VOC detection by $>10 \%$.

Although the most prevalent VOCs worldwide harbor N501Y, this mutation is not present in all variants (10). A PCR-based algorithm for identifying VOCs that use N501Y as the initial screening target must acknowledge this limitation. Given the rapid emergence of new variants, ongoing surveillance is key, and laboratories considering a PCRbased algorithm would need to adapt the algorithm as VOC prevalence changes. For example, our initial screening PCR targeted N501Y, but because of rising rates of B.1.1.7, we adjusted our laboratorydeveloped test to include N501Y and delHV69/70 in a duplexed assay.

PCR-based methods for rapid VOC detection should not replace broader VOC surveillance with WGS, which enables identification of non-N501Y VOCs and can characterize emerging mutations in known VOCs. This ability is critical for enabling laboratories to revise their PCR targets in an ongoing manner to keep pace with local VOC circulation.

In summary, our implementation of an rRTPCR-based algorithm enabled identification of the most common VOCs to date (B.1.1.7, B.1.351, and B.1.1.28/P.1) within 24 hours. This method enables laboratories to perform VOC testing on all SARSCoV-2-positive samples, enhancing VOC surveillance capacity to support near real-time decision making for interrupting transmission.

This article was preprinted at https:/ / www.medrxiv.org/ content/10.1101/2021.03.04.21252928v1.

\section{Acknowledgments}

We are grateful to our medical laboratory technologists, who are highly committed to patient care and laboratory quality improvement. We also acknowledge contributions from John Harding and Althea Hayden and the BCCDC Public Health Laboratory (Natalie Prystajecky, Agatha Jassem, Catherine Hogan, Linda Hoang, and Mel Krajden). 
This work was supported by COVID-19 rapid response grants from GenomeBC (COV-115 to Z.L.B., C.F.L.; COV-033 to C.J.B.), an Exceptional Opportunities Fund-COVID-19 award from the Canada Foundation for Innovation (C.J.B., C.F.L.), a British Columbia Ministry of Health-Providence Health Care Research Institute COVID-19 Research Priorities Grant (C.J.B., C.F.L.), and Public Health Agency of Canada COVID-19 Immunology Task Force COVID-19 Hot Spots Competition Grant (2021-HQ-000120 to Z.L.B., M.G.R.).

G.R. reports participation as a Roche Diagnostics Sequencing Advisory Panel member. Z.L.B. holds a Scholar Award from the Michael Smith Foundation for Health Research.

\section{About the Author}

Dr. Matic is a medical microbiologist at St. Paul's Hospital in Vancouver and a clinical assistant professor at the University of British Columbia. Her primary research interests include virology diagnostics and laboratory quality improvement.

\section{References}

1. Liu Y, Liu J, Xia H, Xianwen Z, Fontes-Garfias CR, Swanson KA, et al. Neutralizing activity of BNT162b2elicited serum - preliminary report. $2021 \mathrm{Feb} 28$ [cited 2021 Mar 3]. https://www.nejm.org/doi/full/10.1056/ NEJMc2102017

2. Xie X, Liu Y, Liu J, Zhang X, Zou J, Fontes-Garfias CR, et al. Neutralization of SARS-CoV-2 spike 69/70 deletion, E484K and N501Y variants by BNT162b2 vaccine-elicited sera [cited 2021 Mar 3]. https:/ / www.nature.com/articles/ s41591-021-01270-4

3. Galloway SE, Paul P, MacCannell DR, Johansson MA, Brooks JT, MacNeil A, et al. Emergence of SARS-CoV-2
B.1.1.7 lineage - United States, December 29, 2020 January 12, 2021. MMWR Morb Mortal Wkly Rep. 2021;70:95-9. https:/ / doi.org/10.15585/mmwr.mm7003e2

4. European Centre for Disease Prevention and Control. Rapid increase of a SARS-CoV-2 variant with multiple spike protein mutations observed in the United Kingdom. 2020 Dec 20 [cited 2021 Mar 3]. https:/ / www.ecdc.europa.eu/sites/ default/ files/documents/SARS-CoV-2-variant-multiplespike-protein-mutations-United-Kingdom.pdf

5. Sabino EC, Buss LF, Carvalho MPS, Prete CA Jr, Crispim MAE, Fraiji NA, et al. Resurgence of COVID-19 in Manaus, Brazil, despite high seroprevalence. Lancet. 2021; 397:452-5. https://doi.org/10.1016/S0140-6736(21)00183-5

6. World Health Organization. Genomic sequencing of SARS-CoV-2: a guide to implementation for maximum impact on public health. 2021 Jan 8 [cited 2021 Mar 3]. https://www.who.int/publications/i/item/9789240018440

7. Ontario Agency for Health Protection and Promotion (Public Health Ontario), Provincial Infectious Diseases Advisory Committee. Interim guidance for infection prevention and control of SARS-CoV-2 variants of concern for health care settings. 2021 Feb 10 [cited 2021 Mar 3]. https:/ / www.publichealth ontario.ca/-/media/documents/ncov/voc/2021/02/ pidac-interim-guidance-sars-cov-2-variants.pdf?la=en

8. European Centre for Disease Prevention and Control. Risk assessment: risk related to the spread of new SARS-CoV-2 variants of concern in the EU/EEA-first update. 2021 Jan 21 [cited 2021 Mar 3]. https:// www.ecdc.europa.eu/en/ publications-data/covid-19-risk-assessment-spread-newvariants-concern-eueea-first-update

9. Charre C, Ginevra C, Sabatier M, Regue H, Destras G, Brun S, et al. Evaluation of NGS-based approaches for SARS-CoV-2 whole genome characterisation. Virus Evol. 2020;6:a075. https://doi.org/10.1093/ve/veaa075

10. Zhang W, Davis B, Chen S, Sincuir Martinez JM, Plummer JT, Vail E. Emergence of a novel SARS-CoV-2 variant in southern California. 2021 Feb 28. [cited 2021 Mar 3]. https://jamanetwork.com/journals/jama/fullarticle/2776543

Address for correspondence: Marc G. Romney, St. Paul's Hospital, Providence Health Care, 1081 Burrard St, Vancouver, BC V6Z 1Y6, Canada; email: mromney@providencehealth.bc.ca 